\title{
Integrated colloidal quantum dot photodetectors with color-tunable plasmonic nanofocusing lenses
}

\author{
Silke L Diedenhofen, Dominik Kufer, Tania Lasanta and Gerasimos Konstantatos
}

High-sensitivity photodetection is at the heart of many optoelectronic applications, including spectroscopy, imaging, surveillance, remote sensing and medical diagnostics. Achieving the highest possible sensitivity for a given photodetector technology requires the development of ultra-small-footprint detectors, as the noise sources scale with the area of the detector. This must be accomplished while sacrificing neither the optically active area of the detector nor its responsivity. Currently, such designs are based on diffraction-limited approaches using optical lenses. Here, we employ a plasmonic flat-lens bull's eye structure (BES) to concentrate and focus light into a nanoscale colloidal quantum dot (CQD) photodetector. The plasmonic lenses function as nanofocusing resonant structures that simultaneously offer color selectivity and enhanced sensitivity. Herein, we demonstrate the first CQD photodetector with a nanoscale footprint, the optically active area of which is determined by the BES; this detector represents an exciting opportunity for high-sensitivity sensing.

Light: Science \& Applications (2015) 4, e234; doi:10.1038/lsa.2015.7; published online 16 January 2015

Keywords: PbS quantum dots; photodetectors; plasmonic bull's eye structures; plasmonic lens

\section{INTRODUCTION}

Nanostructured photodetectors have attracted tremendous interest in recent years by virtue of their potential to offer novel functionalities and superior performance at a fraction of the cost of traditional bulk single-crystalline photodetectors. ${ }^{1}$ Among the various reported technologies for this purpose, colloidal quantum dot (CQD) photodetectors stand out because of their demonstrated performance, spectral selectivity and facile integration into current CMOS (complementary metal oxide semiconductor) technologies. Spectral bands from the visible $^{2}$ to the short-wave infrared ${ }^{3,4}$ and the mid-infrared ${ }^{5,6}$ can be probed via the employment of differently sized quantum dots or different quantum-confined materials. Various classes of photodetectors have been developed, including photoconductors, ${ }^{3}$ photodiodes ${ }^{4}$ and phototransistors, ${ }^{7}$ and their integration into readout focal plane arrays for imaging applications has been successfully demonstrated., ${ }^{8,9}$

An important aspect of the quest for higher-sensitivity detectors is the volume of the active semiconductor material that composes the photodetector. Noise-generation mechanisms scale with the electrically active area of the detector in the shot noise limit ${ }^{10}$ and with the electrical volume of the detector in the generation-recombination ( $\mathrm{G}$ R) noise limit. ${ }^{10}$ Thus, significant improvement in the signal-to-noise ratio can be achieved by fabricating ultra-small-footprint detectors. However, for practical applications, this must be achieved without sacrificing the optically active area of the detector.

To maintain a large optical area in an ultra-small-footprint photodetector, strong light-matter interaction at the nanoscale is required to achieve light concentration well beyond the diffraction limit. To this end, plasmonic structures have been proposed to facilitate light concentration at the nanoscale and enhance light-matter interactions. ${ }^{1-13}$ Plasmonic structures for the enhancement of absorption and the concentration of light into nanoscale volumes have been studied theoretically ${ }^{14-16}$ and experimentally. ${ }^{17,18}$ Dipole antennas have been demonstrated to enhance the optical cross sections of $\mathrm{Ge}$ nanophotodetectors. ${ }^{19}$ Of particular practical interest is the use of a plasmonic bull's eye structure (BES), that is, a concentric plasmonic grating surrounding a nanohole aperture,${ }^{20,21}$ as such a structure can capture light from a large area and concentrate it into a nanoscale aperture. Extraordinary optical transmission has been demonstrated in these structures as a result of the efficient coupling of incident photons into surface plasmon polaritons, which are guided towards the center of the BES and constructively interfere in the aperture, resulting in high transmission efficiencies. ${ }^{20}$ The beaming of the transmitted light through a subwavelength aperture surrounded by a plasmonic BES has been demonstrated, ${ }^{21}$ as has the modification of the direction and efficiency of the photoluminescence of emitters inside the hole. ${ }^{22,23} \mathrm{Si}^{24}$ and $\mathrm{Ge}^{25}$ bulk or micron-sized photodetectors with plasmonic BESs have exhibited photocurrent enhancement at the plasmonic resonance of the structure compared with their BES-free counterparts. However, the potential of the BES to improve both sensitivity and absolute performance compared with corresponding large-scale detectors has not yet been documented. For bull's eye photodetectors to be considered for practical applications, they should outperform both their small- and large-area counterparts. ${ }^{16}$ Moreover, the use of nanoscale single-crystalline bulk photodetectors imposes another major limitation: the quality of a single-crystalline semiconductor device is dictated by its surface trap states. In the case of a 
nanoscale volume of the active semiconductor, the overall device performance is limited by the large surface-to-volume ratio and the correspondingly high density of surface trap states.

We propose that colloidal quantum dots are promising candidates for use as the active semiconducting medium to allow for the exploitation of the benefits of nanoscale photodetectors. In contrast to singlecrystalline semiconductors, the performance of a CQD photodetector is dominated by trap states on the surfaces of the quantum dots, ${ }^{26}$ at a scale that is much smaller (subnanometer scale) than the nanoscale volume and is defined by the nanostructuring of the plasmonic structure (on the order of $100 \mathrm{~nm}$ ). Therefore, the optoelectronic performance of the detector does not deteriorate upon nanopatterning. We demonstrate true performance enhancement in CQD photodetectors compared with both their nanoscale counterparts and large-area detectors based on plasmonic nanofocusing by plasmonic BESs. The resonance of the plasmonic BES depends on its design and is tunable throughout the visible and near-infrared spectral ranges, rendering these photodetectors promising candidates for high-sensitivity image sensor arrays, spectroscopy and other narrow-wavelength-range and color-sorting applications.

\section{MATERIALS AND METHODS}

In this study, $\mathrm{PbS}$ quantum dots with an exciton peak at a wavelength of $950 \mathrm{~nm}$ were synthesized following Ref. 27. After synthesis, butylamine was added to the quantum dot solution, and the solution was stored for three days. Before the devices were fabricated, the oleic acid was removed via precipitation/dispersion in first methanol/toluene and then methanol/octane.

The synthesis of $\mathrm{PbS}$ quantum dots with an exciton peak at $1150 \mathrm{~nm}$ was performed in a similar manner. In that case, we pumped $2 \mathrm{mmol}$ of $\mathrm{PbO}$ and $12 \mathrm{mmol}$ of oleic acid overnight at $85{ }^{\circ} \mathrm{C}$. Afterward, we added $15 \mathrm{~mL}$ of ODE (Octadecene) and raised the temperature to $135{ }^{\circ} \mathrm{C}$. We quickly injected $1 \mathrm{mmol}$ of Tris(trimethylsilyl)silane mixed with $10 \mathrm{~mL}$ of ODE once the required temperature had been reached. The heating was terminated without removing the heating mantle, and the reaction was allowed to cool slowly. We isolated the nanocrystals through the addition of acetone and ethanol $(5: 1)$ and centrifugation; we then purified the nanocrystals via successive dispersion in toluene and precipitation with acetone/ethyl alcohol $(2: 1)$, and we ultimately dispersed them in anhydrous toluene. We also added butylamine to this quantum dot solution and performed a ligand exchange from oleic acid to butylamine. We applied this ligand exchange for both types of quantum dots to reduce the spacing between them during film formation, as butylamine is a much shorter ligand than oleic acid. The absorption spectra of the quantum dots of various sizes that were ultimately used are displayed in Supplementary Fig. S2; they appear as continuous absorption spectra at wavelengths shorter than the exciton peak.

We fabricated plasmonic bull's eye photodetectors on $200 \mathrm{~nm} \mathrm{SiO}$ on top of an Si substrate. We evaporated 5-nm Ti and 100-nm Au onto each sample, and we prepatterned the samples via optical lithography and subsequent etching in aqua regia for the fabrication of the bond pads and the wires connecting the devices. The BESs were fabricated in two stages using electron-beam lithography. In the first stage, the Au triangles were defined, and an evaporation of $50 \mathrm{~nm}$ of $\mathrm{Au}$ and a lift-off process were performed; in the second, the arcs were defined. This step was again followed by the evaporation of 50$\mathrm{nm} \mathrm{Au}$ and a lift-off process. In a third lithography step, the position of the quantum dot layers was defined in the PMMA (polymethyl methacrylate). Butylamine-capped $\mathrm{PbS}$ quantum dots were dissolved in octane with a concentration of $20 \mathrm{mg} \mathrm{mL}^{-1}$. This solution was spin cast onto the PMMA-coated devices for $1 \mathrm{~min}$ and then placed in methanol for $10 \mathrm{~min}$ to remove the butylamine ligands. Removing the butylamine ligands in this manner results in shorter spacing between the quantum dots and has been demonstrated to result in better charge transport. ${ }^{3}$ This process was repeated a second time, after which the sample was placed in acetone for $12 \mathrm{~min}$ at $55{ }^{\circ} \mathrm{C}$ to remove the PMMA and, consequently, the quantum dots on top of the PMMA such that only the quantum dots in the center of the BES remained on the sample. When the $\mathrm{PbS}$ quantum dots with an exciton peak at $1150 \mathrm{~nm}$ were used, the sample was stored overnight in a bath of $60-\mu \mathrm{L}$ dimethoxy-(3-mercaptopropyl)-methylsilane in $10-\mathrm{mL}$ isopropanol before the quantum dots were spin cast.

The transmission was measured by illuminating the fabricated devices on glass microscope slides with collimated light from a halogen lamp. The transmitted light was measured from beneath the central hole of the BES through an immersion oil objective (numerical aperture $=1.4$ ). A pinhole with a diameter of $100 \mu \mathrm{m}$ was used to further reduce the area from which the transmitted light was captured, and the light was sent to a spectrometer.

The finite-difference time-domain simulation geometry was as follows: a total-field scattered-field source was placed around the BES such that the bull's eye was illuminated with a linearly polarized, collimated light beam. We exploited the symmetry of the structure and performed the simulation over only one-fourth of the simulation volume. The simulation volume was terminated in all directions with perfectly matched layers. To simulate the absorption of the light in the $\mathrm{PbS}$ quantum dots, we determined the electric field distribution in the PbS layer and calculated the absorbed power as follows: $P_{\mathrm{abs}}=$ $0.5 \omega E^{\prime} \varepsilon^{\prime \prime} / P_{\text {in }}$, where $\omega=2 \pi c / \lambda$; here, $\lambda$ is the wavelength of the incident light and $E, \varepsilon^{\prime \prime}$ and $P_{\text {in }}$ are the electric field intensity, the imaginary part of the permittivity and the incident field, respectively. The refractive index of the PbS layer was determined as described in Ref. 28.

To measure the spectral photocurrent, we connected the bond pads on the samples to a chip carrier via wire bonding. This chip carrier was placed in a Faraday cage to shield environmental noise. We determined the photocurrent by illuminating each device with spectrally filtered and collimated light from a supercontinuum light source (SuperKExtreme EXW-4; NKT Photonics, Birkerød, Denmark) in the wavelength range from $550 \mathrm{~nm}$ to $1400 \mathrm{~nm}$. The current through the device was measured continuously with an integration time of several milliseconds using a semiconductor parameter analyzer (B1500A; Agilent, Santa Clara, CA, USA), while the device was illuminated by switching the light source on and off and holding for several seconds in each state. Illuminating the device for several seconds allowed the photocurrent in the device to stabilize and yielded a significant number of data points, where each data point corresponded to the integration time of the semiconductor parameter analyzer. At every on- and offstate, several data points were measured to be sure that the photocurrent was stable. In this experiment, the output of the supercontinuum light source could be considered to be continuous, as the response time of the photodetector was much slower than the ultrashort light pulses from the light source. In this manner, the dark current and photocurrent of the device were determined. Because the diameter of the incident light was $\sim 2 \mathrm{~mm}$ and was therefore much larger than the size of our devices $(\sim 6 \mu \mathrm{m})$, and furthermore, because the intensity distribution within the beam was not homogeneous, we performed our quantitative measurements as follows. We performed single-wavelength measurements at $640 \mathrm{~nm}$ and $760 \mathrm{~nm}$, in which we rotated a diffusive plate in front of the device to homogenize the intensity distribution and 
we added a pinhole in front of the sample to achieve a beam diameter of $\sim 6 \mathrm{~mm}$ on the device. Because of the homogeneity of the intensity along the beam profile, we were able to precisely determine the power density incident on the device. All spectral measurements were normalized to the responsivity obtained using this method. In our measurement set-up, the increase in the spot size from $2 \mathrm{~mm}$ to $6 \mathrm{~mm}$ corresponded to an increase in the angle of incidence from normal incidence to $2^{\circ}$. Because the surface plasmon polaritons excited by the plasmonic BES arise through constructive interference, the coupling of light at angles of incidence larger than normal incidence is less efficient than that of light at normal incidence (Supplementary Fig. S3). Therefore, the responsivity and detectivity determined in this manner were, in fact, slightly underestimated.

\section{RESULTS AND DISCUSSION}

The plasmonic BES-PDs (bull's eye structure photodetectors) were fabricated via electron-beam lithography in two stages and the subsequent evaporation of $\mathrm{Au}$, followed by a lift-off process. A third electron-beam lithography step was employed to define the area in which the quantum dots were to be deposited. After the third electronbeam lithography step, the quantum dots were spin coated onto the sample, followed by a third lift-off step to localize the quantum dots at their predefined position. Au was chosen as the plasmonic medium because it forms an ohmic contact with $\mathrm{PbS}$ quantum dots, resulting in a photoconductive configuration with gain. Figure 1a presents a scanning electron micrograph of an Au BES on top of $\mathrm{SiO}_{2}$. The BES consisted of four Au triangles placed $\sim 100 \mathrm{~nm}$ apart. Concentric arcs were placed on top of these triangles. We chose a design consisting of four Au triangles because it is fully symmetric and therefore polarization-independent and because it offers the possibility for lateral electronic contact. The left and right triangles were connected via wires to bond pads for electronic measurements. The quantum dot layer, with a diameter of $\sim 320 \mathrm{~nm}$, is highlighted in the image for clarity. The inset in Figure 1a is a tilted-view scanning electron micrograph showing the complete infiltration of the quantum dots into the gap between the arms of the bull's eye. Atomic force microscopy was utilized to determine the height of the quantum dot layer as well as the groove depth, as shown in the three-dimensional atomic force micrograph presented in Figure 1b. From a cut along the black dashed line indicated in the inset of Figure 1b, the depth of the groove was determined to be $\sim 60 \mathrm{~nm}$, and the height of the PbS quantum dots was determined to be $110 \mathrm{~nm}$ at the edge and $80 \mathrm{~nm}$ in the center on top of the Au. These dimensions were optimized for a BES with a pitch of $700 \mathrm{~nm}$ and were used for the fabrication of BESs with pitches of $600 \mathrm{~nm}, 700 \mathrm{~nm}$ and $800 \mathrm{~nm}$. Additionally, we fabricated a BES with a pitch of $1150 \mathrm{~nm}$. The groove depth in that case was $140 \mathrm{~nm}$, in accordance with the optimum structure determined through simulations. Figure 1c presents a schematic illustration of the BES, in which the parameters used in this manuscript are defined.

To assess the performance improvement achieved in the bull's eye photoconductor, we fabricated two different reference structures on the same sample. In one reference structure, the diameter of the PbS layer was similar to that of the outer arc of the BES (large reference) and was contacted with two Au arms. This reference had the same optically active area as the outer arc of the BES-PD. The other reference contained the same Au triangles as the BES and the same volume of $\mathrm{PbS}$ quantum dots, but no concentric arcs (small reference). This reference had the same electrically active area as the BES-PD. Scanning electron micrographs of these structures are presented in Figure 1d and 1e.
The resonances of the BES were experimentally probed via optical transmission measurements and were compared with the results of simulations performed using a commercial-grade simulator based on the finite-difference time-domain method. ${ }^{29}$ Figure $2 \mathrm{a}$ presents the transmission measurements for BESs without $\mathrm{PbS}$ quantum dots with pitches of $600 \mathrm{~nm}, 700 \mathrm{~nm}$ and $800 \mathrm{~nm}$ and for the small reference. A small maximum was observed at $\sim 500 \mathrm{~nm}$ for all measured devices. This maximum can be attributed to an increase in the transmission at short wavelengths that was further attenuated at shorter wavelengths because of the increasing absorption of Au. The bull's eye resonance in the transmission exhibited a redshift with increasing pitch, in good agreement with the simulations (Figure $2 \mathrm{~b}$ ). The discrepancy between the amplitudes of the measured and simulated transmission can be attributed to two differences between the simulations and the measurements. First, there were differences in the measured and simulated layouts. In the measurement set-up, only the light that was transmitted through the central hole was measured with a high-numerical-aperture objective, whereas in the simulation, all light transmitted through the BES, including the light that passed through the gaps between the $\mathrm{Au}$ triangles, was picked up by the transmission monitor. Second, in the simulated layout, the structure conformed to the ideal design, with sharp edges and flat $\mathrm{Au}$ film. In the measured layout, rounded edges were present because of the lithographic fabrication, along with $\mathrm{Au}$ grains that may have caused wavelength-dependent light scattering. Therefore, the pronounced peak at $500 \mathrm{~nm}$ that appeared in the simulations for all devices was more pronounced in the simulation than in the measurement, where light scattering and less efficient light guiding both played a role. However, even though the simulated layout did not exactly represent the measured layout, the simulations effectively predicted the position of the resonance, demonstrating that finite-difference time-domain simulations are a powerful tool for the design of BES detectors. Because of their sharp resonances in transmission, BESs are of interest for use as tunable-color band-pass filters, in which the spectral sensitivity of the detector is determined by the resonance of the BES and the absorption of the active semiconducting material. The resonance of the BES can then be readily tuned by varying the geometrical parameters of the grating.

To estimate the absorption enhancement in a nanoscale $\mathrm{PbS}$ quantum dot solid placed in the hole of the BES, we performed finite-difference time-domain simulations in which we compared the absorption in the $\mathrm{PbS}$ quantum dot volume in the presence and absence of metallic grooves. The absorption enhancement in the quantum dots is depicted in Figure 2c for bull's eyes structures with pitches of $600 \mathrm{~nm}, 700 \mathrm{~nm}, 800 \mathrm{~nm}$ and $1150 \mathrm{~nm}$, indicating enhancements of up to a factor of 30 at the resonance of the BES $(\lambda=1240 \mathrm{~nm}, p=1150 \mathrm{~nm})$. The electric field intensity along the $x-z$ plane through the center of the BES with a pitch of $700 \mathrm{~nm}$ is presented in Figure 2d for a wavelength of $750 \mathrm{~nm}$, which is on resonance with the pitch of the BES, and in Figure 2e for a wavelength of $605 \mathrm{~nm}$, which is off resonance with respect to the BES. There is a strong difference between the electric field intensity in the center of the structure and that in the PbS quantum dot layer, as is even more visible in the insets of both figures.

To determine the resonance of the BES-PDs optoelectronically, we applied a voltage of $1 \mathrm{~V}$ between the left and right Au triangles of the $\mathrm{BES}-\mathrm{PD}$ and measured the current through the $\mathrm{PbS}$ quantum dots. To account for the wavelength-dependent absorption of the $\mathrm{PbS}$ quantum dot layer, we divided the photocurrent measurements of the BES-PD by the photocurrent measured in the large reference structure for the BES-PDs with pitches of $600 \mathrm{~nm}, 700 \mathrm{~nm}$ and 

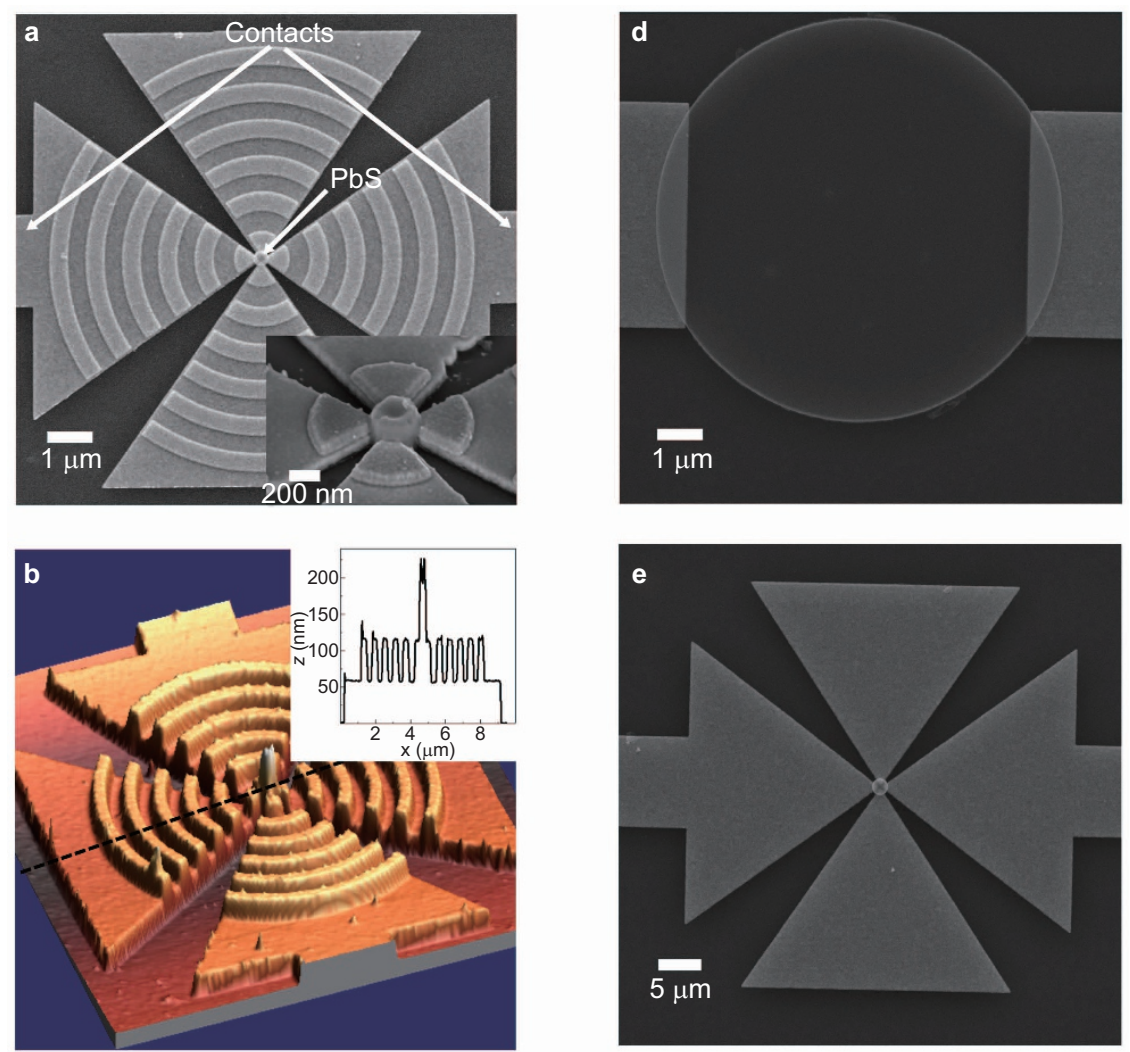

c

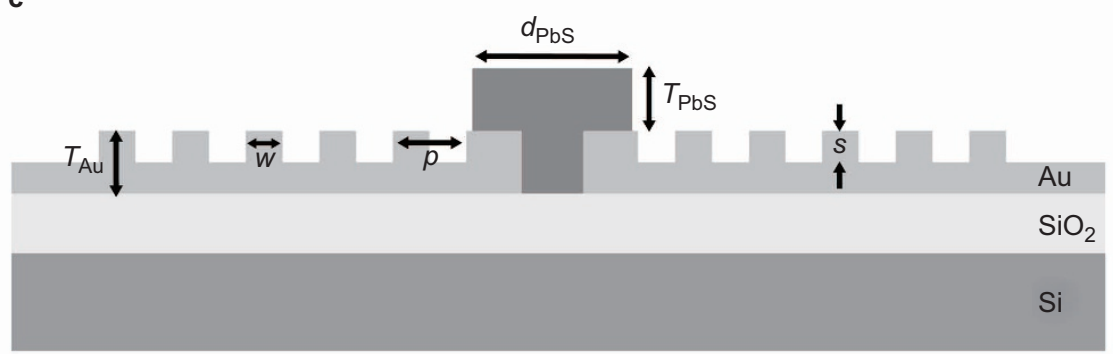

Figure 1 (a) Scanning electron micrograph of a bull's eye structure. The PbS quantum dots in the center and the contact arms at the left and right are indicated. The inset shows a tilted-view scanning electron micrograph highlighting the infiltration of the quantum dots between the Au triangles. (b) 3D atomic force micrograph of the bull's eye structure. The inset shows a line profile collected along the black dashed line indicated in the 3D image. (c) Schematic illustration of the bull's eye structure, on which are indicated the definitions of the groove depth $(s)$, groove width $(w)$, pitch $(p)$, gold thickness $\left(T_{\mathrm{Au}}\right)$, PbS thickness $\left(T_{\mathrm{PbS}}\right)$ and PbS diameter $\left(d_{\mathrm{PbS}}\right)$. (d and $\left.\mathbf{e}\right)$ Scanning electron micrographs of the large reference (d) and the small reference (e). 3D, three-dimensional.

$800 \mathrm{~nm}$ and by the photocurrent measured in the small reference structure for the BES-PD with a pitch of $1150 \mathrm{~nm}$, and we normalized this quantity to its maximum value. The normalized photocurrent enhancement is presented in Figure $2 \mathrm{f}$. For all four measured pitches of $600 \mathrm{~nm}, 700 \mathrm{~nm}, 800 \mathrm{~nm}$ and $1150 \mathrm{~nm}$, the resonance was clearly visible and underwent a redshift with increasing pitch. We observed an enhancement of about $60 \%-80 \%$ in the normalized photocurrent when measured both on resonance $(\lambda=740 \mathrm{~nm}, p=700 \mathrm{~nm})$ and off resonance $(\lambda=660 \mathrm{~nm}, p=700 \mathrm{~nm})$.

To assess the performance enhancement of the BES-PD, we measured the responsivity of the three structures depicted in Figure 1: the BES-PD structure with a pitch of $600 \mathrm{~nm}$, the small reference and the large reference. In all cases, the optically active areas of the detectors were assumed to be identical and equal to the BES area. In the small reference, the electrical volume of the semiconductor material was the same as that in the BES-PD. The optically active area of the detector in the large reference, determined by the $\mathrm{PbS}$ quantum dot film, was the same as the optically active area of the BES-PD, determined by the area of the BES. In Figure 3a, we plot the responsivity of the three photodetectors as a function of the estimated optical intensity in the active volume of the PbS CQDs. The responsivity of a PbS CQD photodetector is known to depend strongly on the incident optical intensity, an effect that is related to the occupation of the sensitizing trap states; ${ }^{2}$ at low optical intensities, long-lived trap states dominate, leading to high optical gain. At high optical intensities, these long-lived trap states are saturated, thereby leading to a progressive reduction in gain. Thus, to fairly compare the performance improvement achieved by virtue of the optical enhancement provided by the BES, we must account for the optical power incident on the PbS layer. We estimated the optical power in the $\mathrm{PbS}$ layer by multiplying the measured incident optical 

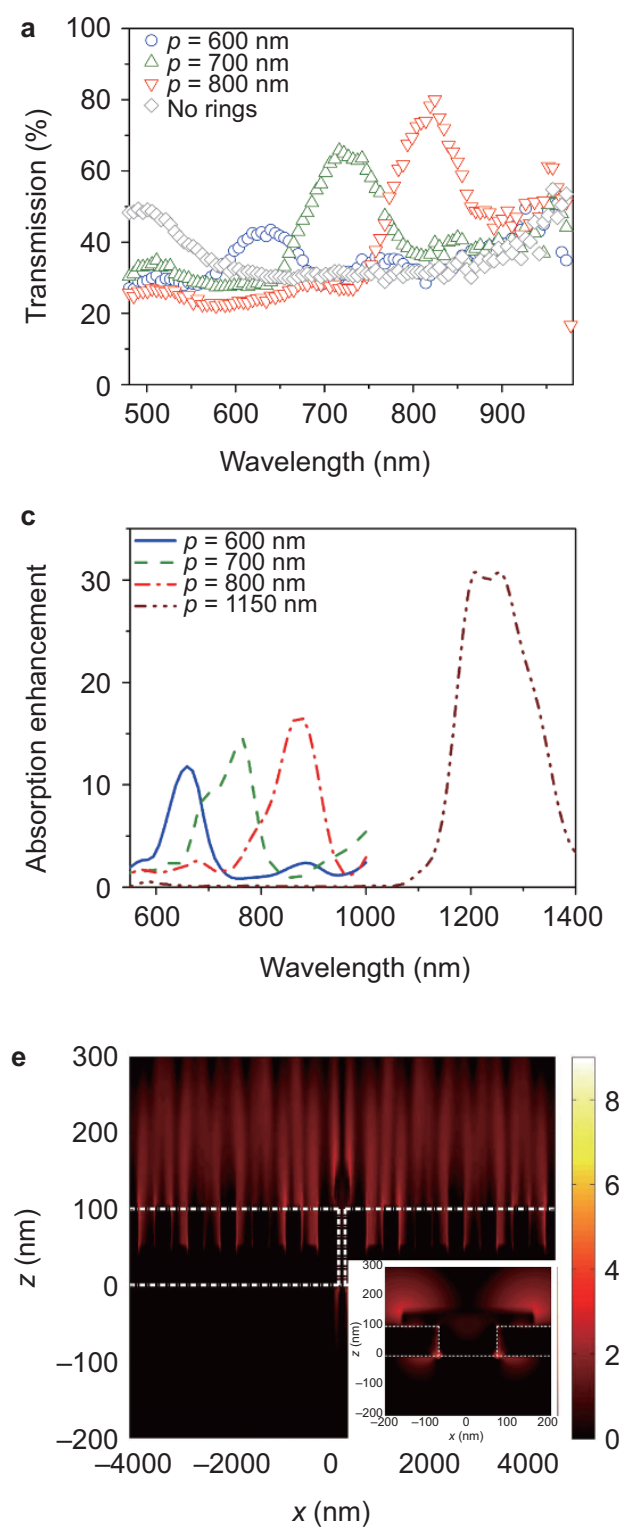
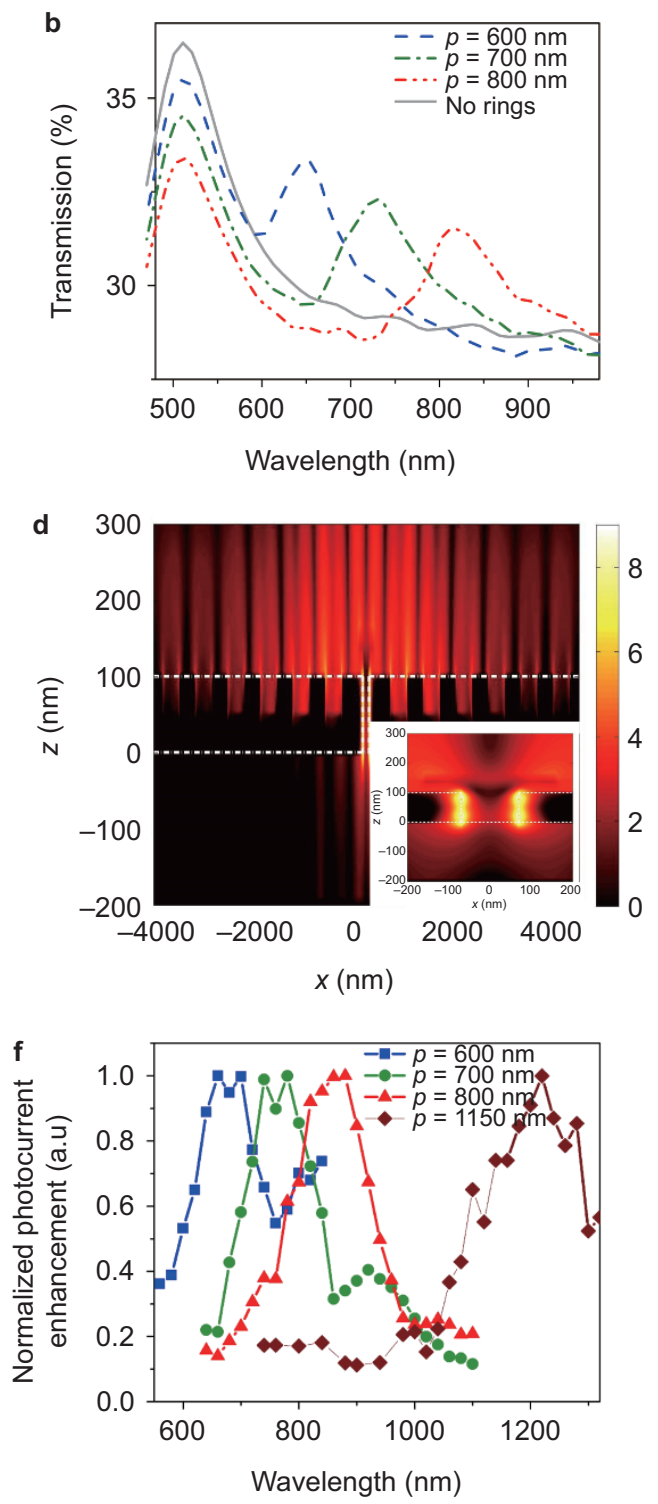

Figure 2 (a) Measured transmission as a function of wavelength for bull's eye structures with pitches of $600 \mathrm{~nm}, 700 \mathrm{~nm}$ and $800 \mathrm{~nm}$ and for the small reference. (b) Simulated transmission for the same structures as in a. (c) Simulated absorption enhancement in a nanovolume of PbS quantum dots in the center of each bull's eye structure compared with the same structure without concentric arcs. (d and $\mathbf{e}$ ) Electric field intensity along the $x-z$ plane through the center of the bull's eye structure in a logarithmic scale for the bull's eye structure with a pitch of $700 \mathrm{~nm}$, at wavelengths both on resonance (755 nm) (d) and off resonance (605 $\mathrm{nm}$ ) (e). The insets present higher-magnification images of the center of the bull's eye structure. The white dashed lines indicate the bottom and top of the Au layer and the grooves. (f) Ratio of the photocurrent measured in the bull's eye structure to that measured in the large reference structure, normalized to the maximum value.

power by a factor calculated from the absorption enhancement at the resonance of the BES determined from the finite-difference time-domain simulations. The responsivity, expressed in units of $\mathrm{A} \mathrm{W}^{-1}$, was calculated as the ratio of the photocurrent over the power incident on the optically active area of the detector (considered, in all cases, to be equal to the area of the large reference detector). The range of optical power intensities presented in Figure 3a was limited by the optical and electronic capabilities of our experimental apparatus. The highest estimated incident optical power corresponds to the maximum laser power available in our set-up. The lowest estimated incident optical power was determined by the electronic noise of our readout system. Photocurrents with lower powers could not be measured, as they could not be differentiated from the dark current. As expected, under the assumption of the same optically active area for the two cases (i.e., the area of the BES), the BES-PD demonstrated a sixfold performance improvement with respect to its small-reference counterpart by virtue of the optical focusing provided by the presence of the metal grating. At the same time, the responsivity of the BES-PD exhibited the same intensity dependence as the large reference, indicating efficient light coupling from the BES to the nanohole.

To demonstrate that the photocurrent enhancement in the BES-PD compared with the small reference can indeed be attributed to the plasmonic resonance of the BES, we present in Figure $3 \mathrm{~b}$ the responsivities of the BES-PDs $(p=600 \mathrm{~nm}$ and $p=1150 \mathrm{~nm})$ and the small reference photodetectors. The measurements of the BES-PD with $p=$ $600 \mathrm{~nm}$ and its reference were acquired at the same estimated incident 

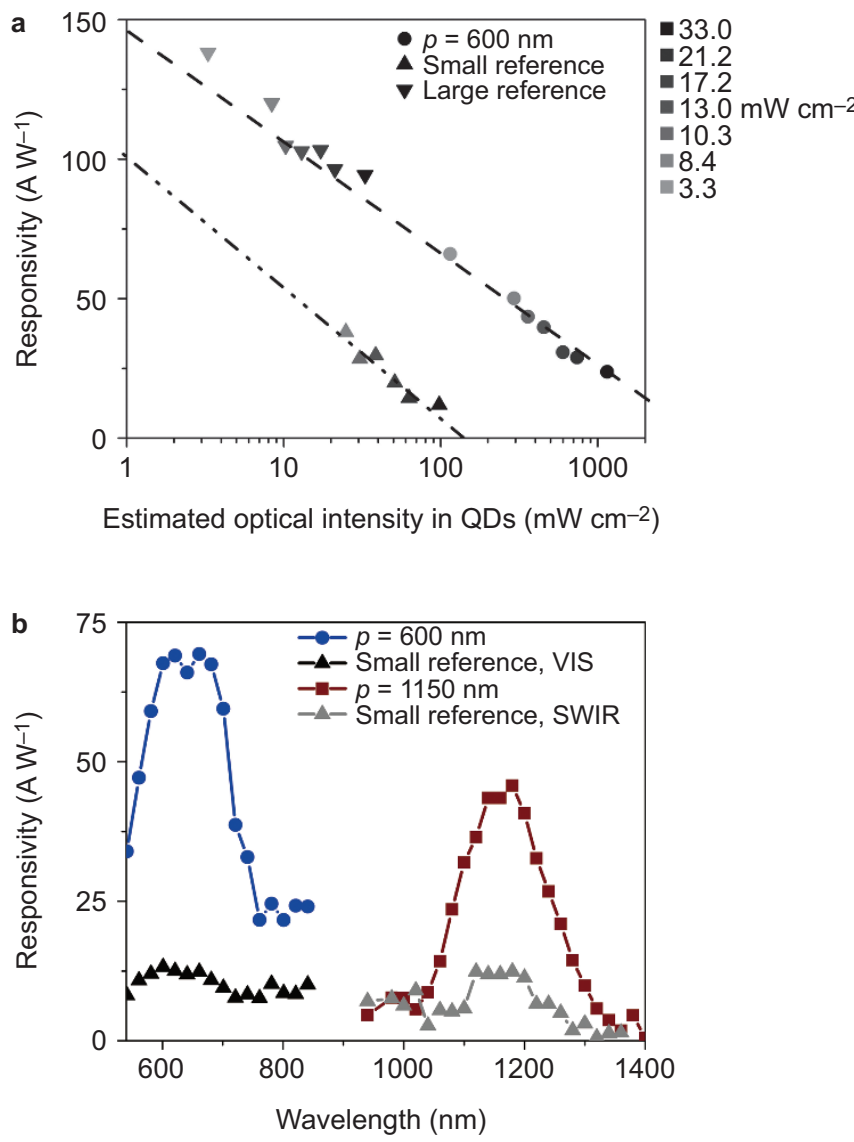

Figure 3 (a) Responsivity as a function of estimated optical intensity in the quantum dot layer, accounting for the concentration of light in the bull's eye structure. The color scale represents the optical power density. (b) Responsivity as a function of wavelength for bull's eye photodetectors with pitches of $600 \mathrm{~nm}$ and $1150 \mathrm{~nm}$ and their corresponding references without concentric arcs. The responsivities of the bull's eye photodetector with $p=600 \mathrm{~nm}$ and its reference were determined at an estimated power of $\sim 4 \mathrm{pW}$. The bull's eye photodetector with $p=1150 \mathrm{~nm}$ and its reference were measured at an optical power of $400 \mathrm{pW}$. QD, quantum dot; SWIR, short-wave infrared; VIS, visible.

optical power of $\sim 4 \mathrm{pW}$, whereas the measurements of the BES-PD with $p=1150 \mathrm{~nm}$ and its reference were acquired at an optical power of $\sim 400 \mathrm{pW}$.

In all cases, the applied electric bias was adjusted to yield the same electric field in all photodetectors $\left(73 \mathrm{kV} \mathrm{cm}^{-1}\right)$ and similar dark current densities $\left(78.2 \mathrm{~mA} \mathrm{~cm}^{-2}\right.$ for the BES-PD with $p=600 \mathrm{~nm}$, $64 \mathrm{~mA} \mathrm{~cm}^{-2}$ for the small reference and $77.6 \mathrm{~mA} \mathrm{~cm}^{-2}$ for the large reference with small $\mathrm{PbS}$ quantum dots). The dark current density of the BES-PD with $p=1150 \mathrm{~nm}$ was $108 \mathrm{~mA} \mathrm{~cm}^{-2}$, and that for its reference was $94.2 \mathrm{~mA} \mathrm{~cm}^{-2}$. As a cross-check, we measured the dark currents of devices without quantum dots. The dark current densities of these devices were within the noise floor of our measurement system and could not be measured, and no photoresponse was detected, indicating that the charges were indeed generated in the quantum dots. Notably, the dark current levels were similar for the BES-PD with $p=600 \mathrm{~nm}$ and its small reference (22 pA and $18 \mathrm{pA}$, respectively), whereas the dark current of the large reference was $388 \mathrm{pA}$. This difference in dark current had a significant impact on the sensitivity performance of these detectors. The figure of merit of a photodetector

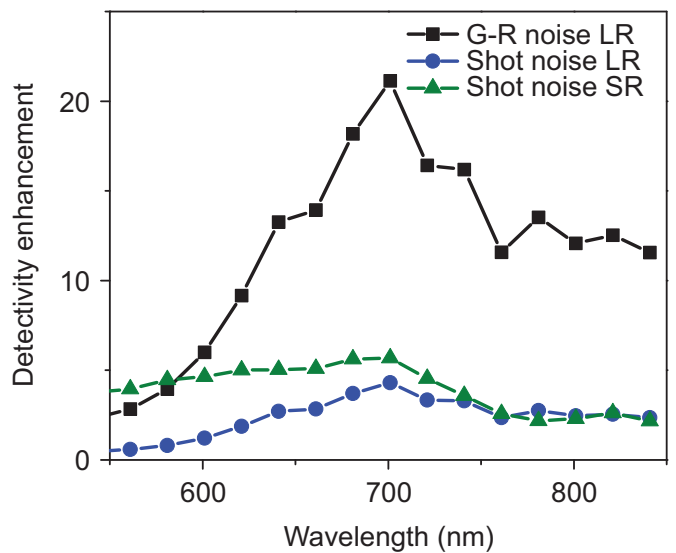

Figure 4 Detectivity enhancement of the bull's eye photodetector compared with the $L R$, determined by assuming either shot noise or $G-R$ noise, and detectivity enhancement of the bull's eye structure compared with the SR, determined by assuming shot noise. G-R, generation-recombination; LR, large reference; SR, small reference.

is the normalized detectivity $D^{*}$, which is defined as

$$
D^{*}=\frac{R \cdot \sqrt{A_{\mathrm{d}} \Delta f}}{I_{\mathrm{N}}}
$$

where $R, A_{\mathrm{d}}$ and $I_{\mathrm{N}}$ are the responsivity, area and noise current, respectively, of the photodetector and $\Delta f$ is its bandwidth. In the shot noise limit, the noise current of a detector is defined by $I_{\mathrm{N}}=\sqrt{2 q I_{\mathrm{D}}}$, where $q$ is the charge of an electron and $I_{\mathrm{D}}$ is the dark current of the detector. We chose to analyze the normalized detectivity in the shot noise limit first, as previous studies have demonstrated that the noise current of CQD photodetectors can reach within $3 \mathrm{~dB}$ of the shot noise limit. ${ }^{3}$ However, because this approach might lead us to underestimate the noise in our detector, at a later stage, we also considered $\mathrm{G}-\mathrm{R}$ noise as the primary noise mechanism. By considering the metallic structure to be part of the detector, we can consider the same active area for both the BES-PD and the small reference detector. In doing so, we determined the normalized detectivities $D^{*}$ of both detectors in the shot noise limit: $1.1 \times 10^{13}$ Jones for the BES-PD with $p=600 \mathrm{~nm}$ and $2.1 \times 10^{12}$ Jones for the small reference detector (assuming an on-resonance wavelength of $640 \mathrm{~nm}$ for the BES-PD and taking the responsivity in the $\mathrm{PbS}$ layer at the same estimated incident optical power of $\sim 4 \mathrm{pW})$. The normalized detectivity $D^{*}$ of the BES-PD with $p=1150 \mathrm{~nm}$ was $1.7 \times 10^{13}$ Jones at a wavelength of $1180 \mathrm{~nm}$, three times higher than that of its reference, which yielded a $D^{*}$ of $4.9 \times 10^{12}$ Jones. In Figure 4, the detectivity enhancement in the shot noise limit is presented as a function of wavelength, demonstrating a fivefold sensitivity improvement in the BES-PD compared with the small reference for a pitch of $600 \mathrm{~nm}$.

To fairly compare the BES-PD structure with the large reference, we would, ideally, consider the responsivities at the same estimated incident optical intensities. Because this task was beyond our experimental capabilities, we chose to consider the responsivities measured at the closest possible estimated incident intensities, a condition that would underestimate the performance of the BES-PD. In Figure 4, we plot the on-resonance enhancement in the normalized detectivity of the BESPD compared with the large reference detector in the shot noise limit. A fourfold enhancement is evident at $700 \mathrm{~nm}$.

Apart from shot noise, photodetectors suffer from G-R noise. This noise component is associated with random generation-recombination events in the bulk of the semiconductor and is dominant for 
infrared detectors and detectors with gain. The G-R noise current is given by

$$
I_{\mathrm{N}}=2 q G \sqrt{A_{\mathrm{d}, \mathrm{e}} \Delta f\left(\eta E_{q}+g_{\mathrm{th}} l_{\mathrm{z}}\right)}
$$

where $q$ and $G$ are the charge of an electron and the photoconductive gain, respectively; $A_{\mathrm{d}}$ e is the electrically active area of the photodetector; $\Delta f$ is its bandwidth; and $\eta, E_{q}, g_{\text {th }}$ and $l_{z}$ are the quantum efficiency, the photo irradiance, the thermal generation of carriers and the detector thickness in the direction of optical generation, respectively. ${ }^{10}$ The suppression of G-R noise requires the fabrication of detectors with smaller electrical volumes; thus, the employment of the BES may result in significant improvements in sensitivity in the G-R noise limit. We thus compared the large-area photodetector and the BES-PD in the G-R noise limit by assuming that all parameters in this equation, except for the electrically active area, were identical for the BES-PD and the large reference. Under this assumption, we can calculate the enhancement of the normalized detectivity as follows

$$
\frac{D_{\mathrm{BE}}^{*}}{D_{\mathrm{Ref}}^{*}}=\frac{R_{\mathrm{BE}}}{R_{\mathrm{Ref}}} \sqrt{\frac{A_{\mathrm{BE}}}{A_{\mathrm{PbS}}}}
$$

where $R_{\mathrm{BE}}$ and $R_{\mathrm{Ref}}$ are the responsivities of the BES-PD and the reference, respectively, and $A_{\mathrm{BE}}$ and $A_{\mathrm{PbS}}$ are the area of the BES-PD defined by the diameter of the outer arc and the area of the $\mathrm{PbS}$ defined by $d_{\mathrm{PbS}}$ (Figure 1c). Figure 4 reveals a normalized detectivity enhancement of a factor of 21 at $700 \mathrm{~nm}$ in the BES-PD compared with the large reference.

\section{CONCLUSIONS}

In summary, we demonstrated that the unique synergy of plasmonic structures with colloidal quantum dots offers a promising pathway toward significant improvement in the sensitivity of colloidal quantum dot photodetectors. We expect further improvements to arise from recent developments in high-quality metallic nanostructures to minimize optical losses. ${ }^{30}$ This work paves the way toward novel functionalities and further performance improvement in other colloidal quantum dot optoelectronic devices, including light-emitting ${ }^{31}$ and solar-harnessing ${ }^{32}$ devices, toward novel color sorting, light management and light beaming applications.

\section{ACKNOWLEDGEMENTS}

We thank M Castro-López for assistance with the transmission measurements, $\mathrm{J}$ Osmond and F Beck for assistance during clean-room fabrication, and M Lundeberg for wire bonding our samples. We acknowledge funding from the European Commission's Seventh Framework Programme for Research under contract PIEF-GA-2011-298596 and from Fundació Privada Cellex Barcelona. We are also grateful to the Ministerio de Ciencia e Innovación for financial support under contract number TEC2011-24744. GK acknowledges MICINN for a Ramon y Cajal Fellowship.

1 Konstantatos G, Sargent EH. Nanostructured materials for photon detection. Nat Nanotechnol 2010; 5: 391-400.

2 Konstantatos G, Clifford J, Levina L, Sargent EH. Sensitive solution-processed visiblewavelength photodetectors. Nat Photonics 2007; 1: 531-534.
3 Konstantatos G, Howard I, Fischer A, Hoogland S, Clifford J et al. Ultrasensitive solution-cast quantum dot photodetectors. Nature 2006; 442: 180-183.

4 Clifford JP, Konstantatos G, Johnston KW, Hoogland S, Levina L et al. Fast, sensitive and spectrally tunable colloidal-quantum-dot photodetectors. Nat Nanotechnol 2009; 4: 40-44.

5 Böberl M, Kovalenko MV, Gamerith S, List EJ, Heiss W. Inkjet-printed nanocrystal photodetectors operating up to $3 \mu \mathrm{m}$ wavelengths. Adv Mater2007; 19: 3574-3578.

6 Keuleyan S, Lhuillier E, Brajuskovic V, Guyot-Sionnest P. Mid-infrared HgTe colloidal quantum dot photodetectors. Nat Photonics 2011; 5: 489-493.

7 Konstantatos G, Badioli M, Gaudreau L, Osmond J, Bernechea M et al. Hybrid graphene-quantum dot phototransistors with ultrahigh gain. Nat Nanotechnol 2012; 7: 363-368.

8 Rauch T, Böberl M, Tedde SF, Fürst J, Kovalenko MV et al. Near-infrared imaging with quantum-dot-sensitized organic photodiodes. Nat Photonics 2009; 3: 332-336.

9 Clifford JP. Colloidal quantum dot Schottky barrier photodiodes. PhD thesis, University of Toronto, Toronto, Canada, 2008.

10 Dereniak EL, Boreman GD. Infrared Detectors and Systems. Hoboken, NJ: Wiley, 1996.

11 Schuller JA, Barnard ES, Cai W, Jun YC, White JS et al. Plasmonics for extreme light concentration and manipulation. Nat Mater 2010; 9: 193-204.

12 Novotny L, van Hulst N. Antennas for light. Nat Photonics 2011; 5: 83-90.

13 Gramotnev DK, Bozhevolnyi SI. Nanofocusing of electromagnetic radiation. Nat Photonics 2014; 8: 13-22.

14 Yu Z, Veronis G, Fan S, Brongersma ML. Design of midinfrared photodetectors enhanced by surface plasmons on grating structures. App/ Phys Lett 2006; 89: 151116.

15 Le Perchec J, Desieres Y, de Lamaestre RE. Plasmon-based photosensors comprising a very thin semiconducting region. Appl Phys Lett 2009; 94: 181104.

16 Bhat RD, Panoiu NC, Brueck SR, Osgood RM. Enhancing the signal-to-noise ratio of an infrared photodetector with a circular metal grating. Opt Express 2008; 16: 45884596.

17 Tang L, Miller DA, Okyay AK, Matteo JA, Yuen Y et al. C-shaped nanoapertureenhanced germanium photodetector. Opt Lett 2006; 31: 1519-1521.

18 Shackleford JA, Grote R, Currie M, Spanier JE, Nabet B. Integrated plasmonic lens photodetector. Appl Phys Lett 2009; 94: 083501.

19 Tang L, Kocabas SE, Latif S, Okyay AK, Ly-Gagnon DS et al. Nanometre-scale germanium photodetector enhanced by a near-infrared dipole antenna. Nat Photonics 2008; 2: 226-229.

20 Thio T, Pellerin KM, Linke RA, Lezec HJ, Ebbesen TW. Enhanced light transmission through a single subwavelength aperture. Opt Lett 2001; 26: 1972-1974.

21 Lezec HJ, Degiron A, Devaux E, Linke RA, Martin-Moreno L et al. Beaming light from a subwavelength aperture. Science 2002; 297: 820-822.

22 Aouani H, Mahboub O, Bonod N, Devaux E, Popov E et al. Bright unidirectional fluorescence emission of molecules in a nanoaperture with plasmonic corrugations. Nano Lett 2011; 11: 637-644.

23 Jun YC, Huang KC, Brongersma ML. Plasmonic beaming and active control over fluorescent emission. Nat Commun 2011; 2: 283.

24 Ishi T, Fujikata J, Makita K, Baba T, Ohashi K. Si nano-photodiode with a surface plasmon antenna. Jpn J Appl Phys 2005; 44: L364-L366.

25 Ren FF, Ang KW, Ye J, Yu M, Lo GQ et al. Split bull's eye shaped aluminum antenna for plasmon-enhanced nanometer scale germanium photodetector. Nano Lett 2011; 11: 1289-1293.

26 Konstantatos G, Levina L, Fischer A, Sargent EH. Engineering the temporal response of photoconductive photodetectors via selective introduction of surface trap states. Nano Lett 2008; 8: 1446-1450.

27 Mihi A, Bernechea M, Kufer D, Konstantatos G. Coupling resonant modes of embedded dielectric microspheres in solution-processed solar cells. Adv Opt Mater 2013; 1 : 139-143.

28 García de Arquer FP, Beck FJ, Konstantatos G. Absorption enhancement in solution processed metal-semiconductor nanocomposites. Opt Express 2011; 21: 2103821049.

29 Lumerical Solutions, Inc. http://www. lumerical.com/tcad-products/fdtd/.

30 Nagpal P, Lindquist NC, Oh SH, Norris DJ. Ultrasmooth patterned metals for plasmonics and metamaterials. Science 2009; 325: 594-597.

31 Shirasaki Y, Supran GJ, Bawendi MG, Bulović V. Emergence of colloidal quantum-dot light-emitting technologies. Nat Photonics 2013; 7: 13-23.

32 Polman A, Atwater HA. Photonic design principles for ultrahigh-efficiency photovoltaics. Nat Mater 2012; 11: 174-177.

This work is licensed under a Creative Commons Attribution-

NonCommercial-ShareAlike 3.0 Unported License. The images or other third party material in this article are included in the article's Creative Commons license, unless indicated otherwise in the credit line; if the material is not included under the Creative Commons license, users will need to obtain permission from the license holder to reproduce the material. To view a copy of this license, visit http://creativecommons.org/licenses/by-nc-sa/3.0/ 